\title{
Assessing treatment effects and publication 2 bias across different specialties in medicine: a large empirical study of the Cochrane ${ }_{4}$ Database of Systematic Reviews
}

5 Simon Schwab ${ }^{1,2, *}$, Giuachin Kreiliger $^{3} \&$ Leonhard Held ${ }^{1,2}$

$6{ }^{1}$ Center for Reproducible Science (CRS), University of Zurich, Switzerland.

$7{ }^{2}$ Epidemiology, Biostatistics and Prevention Institute (EPBI), University of Zurich, Switzerland.

$8{ }^{3}$ Master Program in Biostatistics, University of Zurich.

9 Study protocol: osf.io/3a28k (registered on July 9, 2019)

10 Supplementary information: $\underline{\text { osf.io/dtqs } 5}$

11 STROBE checklist: osf.io/b6gxf

12 *Corresponding author: Simon Schwab, Center for Reproducible Science, University of Zurich,

13 Hirschengraben 84, 8001 Zurich, Switzerland, E-mail: simon.schwab@uzh.ch

14 Abstract: 257 words

15 Main text: 5,650 words 


\section{${ }_{16}$ Abstract}

17 Objectives To compare reporting of statistically significant treatment effects and adverse events

18 across medical specialties, to determine associations of statistical significance with journal metrics,

19 to test small-study effects in meta-analyses (when small studies report more extreme treatments

20 effects than large studies), to assess publication bias in funnel plots and to quantify bias-adjusted

21 treatment effects.

22 Design Large empirical study of treatment effects.

23 Methods We investigated outcomes from 57,186 studies from 1922 to 2019, and overall 99,129 24 meta-analyses and 5,534 large meta-analyses ( $\geq 10$ studies) from the Cochrane Database of 25 Systematic Reviews (CDSR). Descriptive statistics, generalized linear mixed models, 26 regression-based tests, and selection models were used to analyze one of the largest collections of 27 evidence in medicine.

28 Results Medical specialties showed differences in the prevalence of statistically significant results 29 of efficacy and safety outcomes. Altogether 19\% (95\%-CI from $18 \%$ to $20 \%)$ of the large 30 meta-analyses showed evidence for small-study effects, but only 3.9\% (95\%-CI from 3.4\% to 31 4.4\%) showed evidence for publication bias after further assessment of funnel plots. Adjusting 32 treatment effects resulted in overall less evidence for efficacy, and treatment effects in some 33 medical specialties or published in prestigious journals were more likely to be statistically 34 significant while randomized controlled trials (RCTs) were less likely to report a statistically 35 significant effect.

36 Conclusions These results suggest that exaggerated effects from small studies cause greater 37 concern than publication bias. It is problematic when studies that report more extreme results 38 become more visible in prestigious journals instead of studies of the highest quality.

39 Keywords: Cochrane, systematic reviews, meta-analysis, small-study effects, publication bias 


\section{${ }_{40}$ Strengths and limitations of this study}

41 - This is one of the few studies providing a large-scale assessment of treatment effects, small-study effects and publication bias across different medical specialties.

- Many methods are available to assess small-study effects and publication bias, we only considered two methods; however, these were among the most widely used and recommended ones.

- Bias may be underestimated as the methods have low statistical power and only a subset of large meta-analyses were therefore considered; however, these may reflect among the most relevant medical interventions to date as many studies have been performed. 


\section{${ }_{49}$ Introduction}

50 Publication bias is a major concern in clinical research as it affects the combined effects from

51 meta-analyses of intervention studies and distorts the overall evidence for the efficacy of a

52 treatment $[1,2]$. The problem was already characterized more than half a century ago [3] and a

53 number of studies provided compelling evidence for publication bias by following studies from

54 protocol approval until the publication of outcomes [4,5]. The problem originates when studies

55 with a null result are not considered worthy to be written up and submitted by researchers, or may

56 not be treated favorably in peer review; hence, are less likely to be published [6,7]. Moreover,

57 statistically significant studies are more likely to be published in journals with a high citation

58 impact factor [8] and get more citations [9]. Thus, positive results receive more attention than

59 negative results.

60 Many countries and medical journal publishers require trial registration with the aim to prevent

61 publication bias, for example, the International Committee of Medical Journal Editors (ICMJE).

62 Since 2007 the U.S. Food and Drug Administration Amendments Act (FDAAA) demands that

63 interventional clinical trials report their results directly to the US trial registry "ClinicalTrials.gov"

64 within 1 year of the completion of the study; however, recent research showed that compliance with

65 FDAAA 2007 is poor with $59 \%$ of the studies not reporting results in time [10]. The retrieval

66 process of unpublished results can be difficult, time consuming and not always successful. Thus,

67 statistical methods can be useful to not only assess but also adjust meta-analyses for inflated

68 treatment effects and publication bias.

69 In clinical research multiple studies on the same treatment are conducted, and meta-analyses are the

70 established statistical tool to estimate the combined treatment effect. Combined effects are

71 weighted averages of the study effects with effects from large studies having more weight than

72 effects from smaller studies. However, when the chance of publication increases for small studies

73 with large effects, and decreases when there is no effect, then there is a positive association

74 between effect sizes and standard errors of the studies, known as small-study effect [11]. This

75 association is assessed by regression and can be shown as asymmetry in a funnel plot [12], but

76 these methods also have some limitations [13]. 
77 A small number of studies in meta-analyses and heterogeneity across studies can mislead funnel 78 plot based methods. Even though it is often assumed that publication bias may be a plausible 79 explanation for funnel plot asymmetry, there are also other possible causes, for example, low 80 quality studies that report inflated effect sizes, or clinical heterogeneity of patients when small 81 studies focus on high-risk patients for whom the treatment may be more effective. Therefore, it is 82 important to follow guidelines when applying such methods [14,15]. Sterne et al. [14] 83 recommended Harbord's test [16] for dichotomous outcomes using odds ratios, and Egger's test 84 [12] for continuous outcomes. Also the Cochrane handbook recommends the same tests, provided 85 that there are at least 10 studies [17].

86 In contrast to methods based on a funnel plot, selection models explicitly model publication bias.

87 Selection models adjust meta-analytic data by specifying a model that describes the mechanism by 88 which effect sizes may be suppressed. The Copas selection model is among the more sophisticated 89 selection models [18] and investigates whether studies with a certain sample size showing larger 90 effects have a greater probability to enter a meta-analysis. A common feature in both 91 regression-based methods and the Copas selection model [19,20] is that effect estimates can be 92 adjusted for bias. In practice, regression-based adjustments of treatment effects were successful in 93 the prediction of the effect of antidepressant trials from the FDA trial registry using a biased subset 94 of the data [21].

95 Previous research estimated publication bias from the Cochrane Database of Systematic Reviews 96 (CDSR). Sutton et al. [22] estimated with the trim-and-fill method that around 50\% of the reviews 97 had missing studies in a total of 48 Cochrane reviews. A large study [15] investigated 98 meta-analyses with binary outcomes from 1,669 Cochrane reviews and found funnel plot 99 asymmetry in $71(19 \%)$ of the 366 meta-analyses that met inclusion criteria for regression-based 100 tests $(p<.10$, two-sided); on the other hand, another study found no convincing evidence for 101 publication bias [23] but they investigated a small subset of only 83 meta-analyses from the CDSR 102 to compare to a matched sample of meta-analyses from psychology. A study using a selection 103 model in 1,106 meta-analysis in the CDSR found positive findings to be $27 \%$ more likely to be 104 included in a meta-analysis [24]. An extensive and recent study by Lin et al. [25] investigated 105 30,000 meta-analyses from the CDSR and tested various methods among those regression-based 106 tests (but no selection models) and reported considerable funnel plot asymmetry in $20 \%-40 \%$ of 107 the meta-analyses; however, they included meta-analysis with only five studies which is at odds 
108 with the common guideline of at least ten studies. Some methodological studies have been 109 performed with data from the CDSR to evaluate various asymmetry tests and found that Egger's 110 linear regression was most sensitive to detect asymmetry [26,27]. Statistical power can be increased 111 by choosing a more liberal $p$-value threshold with no substantial increase in false positive rate [28], 112 and indeed in many studies a significance level of $p<0.10$ is used.

113 These studies were sometimes constrained to only a subset of the CDSR data or to methodology 114 that is generally not recommended such as trim-and-fill which has been shown to be inferior to 115 regression-based methods (Moreno, Sutton, Ades, et al., 2009). Often it was not reported how 116 outcomes related to efficacy were distinguished from outcomes related to adverse events. This is

117 important because for outcomes related to adverse events, if anything, we would expect publication 118 bias to operate in favor of null results. Furthermore, adjustment of the effect sizes is rarely 119 performed, and to the best of our knowledge, none of the studies compared funnel-plot asymmetry 120 and publication bias across different medical specialties.

121 The four aims of our study were a large-scale empirical assessment of the reported effects and their 122 statistical significance in (1) primary studies and in (2) meta-analyses, (3) the prevalence of funnel 123 plot asymmetry and publication bias, and (4) the magnitude of the adjustment of biased estimates 124 across different specialties in medicine. The hypotheses are specified in more detail in 125 Supplementary Table 1. Our primary data source was the CDSR, the probably largest and most 126 reliable collection of evidence in medicine.

\section{${ }_{127}$ Methods}

128 A study protocol was written and the methods were specified in advance including a statistical 129 analysis plan. The protocol was registered on July 9, 2019 (osf.io/3a28k). Deviations from the 130 protocol are specified at the end of the methods sections. The complete pipeline with analysis code 131 written in $\mathrm{R}$ is available at osf.io/uv397. Even though this is not a systematic-review itself but a 132 meta-research study on meta-analyses from systematic reviews we report this study following the 133 STROBE guidelines [29], the checklist for this study can be found at osf.io/b6gxf. 


\section{Search strategy and study selection}

135 Systematic reviews of the type "intervention" were selected from the CDSR which were 7,931 136 reviews as of November 11, 2019. These reviews assessed the benefits and harms of interventions 137 used in healthcare and health policy and involved primary studies from randomised controlled trials 138 (RCT) as well as observational studies. All studies involved an intervention, for example, a drug, a 139 surgical procedure, a psychotherapy, a device, preventive care, etc.

\section{Data collection}

141 The Cochrane Library conducts systematic reviews and is one of the largest and most reliable 142 sources for evidence in health care. Data from 6,075 (77\%) of all the systematic reviews could be 143 retrieved which comprised 253,430 meta-analyses with outcomes from 71,986 primary studies 144 from over 50 million patients (Figure 1A). The primary studies included the sample sizes and the 145 number of events for both arms when the outcome was dichotomous, or the means and standard 146 deviations when the outcome was continuous. We further extended the dataset by scraping 147 additional information for the 6,075 Cochrane reviews: the full reference of the included primary 148 studies (including the journal name) and the the table "characteristics of included studies" which 149 included additional details on the methods, participants, interventions, and outcomes of the 150 intervention studies.

151 Furthermore, we collected citation information from Google Scholar (May 2020; 152 scholar.google.com) for 40,306 (70\%) and the journal rankings from SCImago (February, 2020; 153 www.scimagojr.com) for 39,270 (69\%) of the primary studies selected for analyses in Figure 1B. 154 Software in $\mathrm{R}$ to fetch and process data from the CDSR and the other sources is available at 155 osf.io/uv397.

\section{Data processing}

157 Beside the outcomes related to the efficacy of an intervention, there were also outcomes related to 158 adverse events (13.5\%), withdrawal/dropout from the study (2.4\%), and bias/sensitivity analyses $159(1.3 \%)$. We identified and categorized the outcomes using regular expressions on the comparison 160 name, outcome name, and subgroup name as provided by the CDSR. 
161 The 53 Cochrane review groups were merged into 19 medical specialties, see Supplementary Table

162 2. Assignment was based on thematic overlap, e.g. various cancer groups into "oncology", various

163 neurological conditions into "neurology".

\section{Inclusion criteria}

165 The data selection process for the analyses conducted is shown in Figure 1B-C. The analysis of the 166 effect sizes (Figure 1B) included all effects from published primary studies. We checked that all the 167 reported effects from the primary study were unique because the CDSR data can include the same 168 effect more than once, for example, when different systematic reviews included the same primary 169 study.

170 For the analysis of the combined effects from meta-analyses the number of primary studies was at

171 least 2 (Figure 1C). Furthermore, we excluded meta-analyses that appeared more than once or that 172 contained the same study effect more than once, for example, when the same meta-analysis was 173 conducted but with different subgroup analyses, or when a meta-analysis contains multiple 174 subgroup analyses that were sensitivity analyses. Meta-analyses based on subgroups were generally 175 included because within the hierarchical structure of the CDSR (comparisons, outcomes and 176 subgroups) systematic reviewers sometimes analyzed what actually were different comparisons as 177 different subgroups (e.g. different comparison of drugs).

178 For the asymmetry tests, selection model analysis, and adjustment of effect sizes (Figure 1D) in the 179 meta-analyses we strictly followed the guidelines by Ioannidis and Trikalinos [15] and used the 180 following four inclusion criteria: (1) the meta-analysis contains $\geq 10$ studies, (2) heterogeneity $I^{2}<$ $18150 \%$, (3) the ratio of the minimum and maximum study variance $>2$, and (4) at minimum one 182 primary study must show a statistically significant effect.

\section{Statistical analyses}

184 We used Wilson confidence intervals for proportions and Wald confidence intervals for odds ratios 185 and coefficients from regression. Differences in proportions across medical specialties were 186 assessed with a Pearson's $\chi^{2}$-test without continuity correction. 


\section{Effect sizes from primary studies}

188 Effect sizes from primary studies included various outcome measures, the most common were risk 189 ratios $(46 \%)$, mean differences $(26 \%)$, odds ratios (11\%), standardized mean differences (SMD) $190(8 \%)$, Peto odds ratios (5\%), risk differences $(1.5 \%)$ and hazard ratios $(1.1 \%)$, others were not 191 considered but the above mentioned represented $98 \%$ of all the primary study effect sizes reported

192 in the CDSR. Comparing reported effect sizes from primary studies across medical specialties 193 required harmonization. We recalculated all effect sizes for the primary studies using escalc () 194 from the R package metafor [30]. For continuous outcomes the SMD (Hedges' $g$ ), for

195 dichotomous outcomes the odds ratios was used. Odds ratios were transformed into Hedges' $g$ 196 which were then transformed into Pearson's $r$ [31]. Statistical significance was calculated with a

197 Wald's test which was performed on the original effect measure as reported in the CDSR (e.g., risk 198 ratio, hazard ratio, etc.); for mean differences we applied a two-sample Student's $t$-test.

\section{Combined effect estimates from meta-analyses}

200 We used the R package meta [32] for conducting meta-analyses for continuous outcomes (Hedges' 201 g) and binary outcomes (odds ratio). The combined effect from the random effects meta-analysis 202 was converted into a Pearson's $r$ [31]. Statistical significance was determined by random effects 203 meta-analyses of the original effect measures as specified in the CDSR.

\section{Asymmetry tests and adjustment of combined effects}

205 Given the vast number of methods that now exist to study publication bias and sometimes lack 206 proper validation [33], we constrained our analyses to three widely used methods, the 207 regression-based Egger's test [12], Harbord's tests [16] and the Copas selection model analysis $208[19,20]$. We used the package meta [32] to detect funnel plot asymmetry in a meta-analysis using 209 the original effect measure as reported in the CDSR with Egger's linear regression; for 210 meta-analyses with odds ratios as effect measure we applied Harbord's test. Meta-analyses of Peto 211 odds ratios were performed with odds ratios, and meta-analysis of hazard ratios or risk differences 212 were performed with risk ratios instead; funnel plots of risk differences are not recommended [17]. 213 All tests were performed one-sided $(\mathrm{p}<0.05)$; the rationale for this will be explained further below.

214 The metasens package [34] was used to adjust combined effects via a regression-based method 215 known as limit meta-analysis [35,36] and the via Copas selection model [19,20]. The adjustments 216 were performed with the log odds ratios for dichotomous outcomes and with the SDMs for 
217 continuous outcomes in order to harmonize and compare results across medical specialties on the 218 Pearson's $r$ scale [31].

219 For the assessment of whether the adjustment increased or decreased the evidence for an 220 intervention we determined the side that favours the treatment as the side where there were more 221 studies with a statistically significant effect. While it is possible in a meta-analysis that a majority 222 of studies do not show evidence for a treatment benefit, it is unlikely that a majority of studies 223 demonstrate a statistical significant effect in the unexpected, opposite direction. Once the 224 anticipated side of the treatment effect was determined, a one-sided regression-based test $(p<0.05)$ 225 could be performed which had the advantage to exclude situations where asymmetry was caused by 226 smaller studies showing a larger effect in the unexpected direction. Thus, the operationalization of 227 small-study effects by means of asymmetry tests was more accurate.

\section{Small-study effect with probable publication bias}

229 Asymmetry tests can discover that smaller studies report stronger evidence for a treatment but do 230 not directly assess publication bias, and it is important to further evaluate funnel plot asymmetry 231 [13]. Thus, we further assessed the funnel plots with asymmetry $(p<0.05)$ and determined the 232 proportion of smaller studies with a significant result vs. a null result in any of the top 50\%, 33\% 233 and $25 \%$ of the studies with the largest standard errors (the smallest studies). If an equal or larger 234 number of studies were found we assume "small study-effects with probable publication bias" as 235 publication bias seems more likely in such situations. On the other hand, when more null results 236 were present in all of the quantiles then publication bias seems less likely, for example, in situations 237 where there is strong evidence for asymmetry but only a minority studies were statistically 238 significant. While publication bias may theoretically still be possible and there are "hidden" studies 239 in the funnel plot, their impact may not be so severe when a majority of the smaller studies that 240 were published show a null result.

\section{Change in evidence for treatment effects}

242 We converted the $p$-values from the combined treatment effects from the fixed effects 243 meta-analysis, from the bias-adjusted regression-based analysis and from the Copas selection 244 model analysis into Bayes factors bounds [37] and categorized those into six levels of evidence 245 from "weak" to "decisive" against the null hypothesis [38]. The change in evidence after 
246 adjustment was determined by comparing the proportion of treatment effects falling in those 247 categories.

\section{Modeling of outcomes: statistical significance, small study-effects and bias adjustment}

249 Six generalized linear mixed-effects (labeled 1-6 in the following text) were performed with the R 250 package lme4 [39] for the binary outcome statistical significance $(p<0.05$, two-sided) of the 251 treatment effects in primary studies (1) and combined effects from meta-analyses (2). We modeled 252 the evidence for asymmetry using the test statistic ( $t$-score) from the regression-based test (3) was 253 well as the binary outcome (4) of whether the asymmetry tests gave a positive result or not ( $p<$ 254 0.05, one-sided). We also modeled the change in Pearson's $r$ after adjustment with regression-based 255 adjustment (5) and Copas adjustment (6). The explanatory variables for model 1 were the medical 256 specialties (19 levels), the journal rank quartile (4 level), the study size, the number of citations per 257 year, the publication year and whether the study was a RCT (binary). The explanatory variables for 258 the models 2-6 were the medical specialties (19 levels), the median journal rank (across studies), 259 the total sample size (across studies), the median number of citations per year (across studies), the 260 review year and the number of studies in the meta-analysis.

261 Explanatory variables that were right-skewed were $\log _{2}$ transformed. Random effects accounted for 262 dependencies and nesting in the data; these were the study id to model results form primary studies, 263 and the Cochrane review id for for the meta-analyses. The models were in more detail specified in 264 the study protocol section 4.3 .

\section{Missing data}

266 There was no missing data for the outcomes variables treatment effect from primary studies, 267 combined treatment effect from meta-analyses, assessment of small-study effect, or adjustment by 268 regression. For adjustments of treatment effects by Copas 143 (2.6\%) meta-analyses were not 269 estimable.

270 We performed complete case analysis with missing data in the explanatory variables used in the six 271 regression models. Missing data were in publication year (14\%), journal rank (31\%), and number 272 of citations $(32 \%)$ with a total of $44 \%$ of the cases excluded for the outcome variable statistical 273 significance of the primary studies. The missing data mainly affected published studies in journals 274 with no journal rank or no citation information available. Google Scholar only provides data if the 275 study has at least a single citation. For the outcome statistical significance of meta-analyses missing 
276 data were in the explanatory variables median journal rank (6\%) and median number of citations

$277(11 \%)$ with a total of $14 \%$ of the cases excluded. For the four remaining regression models with the

278 outcomes publication bias (continuous and binary) and bias adjustments (regression-based and

279 Copas) missing data were in the median journal rank (2\%), and median number of citations $(3 \%)$

280 with a total of $4 \%$ of the cases excluded.

\section{Changes from the initial protocol}

282 We used the binary outcome of whether the effects were statistically significant but did not specify 283 this clearly in the research hypothesis and just used the term "effect size" instead of "statistical 284 significance of effect size" (protocol section 3: 1c and 2c; 4.3: 1c and 2c). We named the regression 285 model for hypothesis 2c but we did not specify the dependent variable in the statistical analysis 286 plan (protocol section 4.3; 2c). We were able to add a binary variable RCT (yes/no), i.e. whether a 287 study was reported as a randomized controlled trial, and used this additional variable in the 288 modeling (protocol section 4.3; 1c). We used categorical quartiles of the journal rank instead of the 289 continuous variable (protocol section 4.3; 1c); these were provided by SCImago themselves. We 290 applied Harbord's test only for odds ratios and not generally for all binary outcomes as specified in 291 the protocol (protocol section 4.4).

\section{Patient and public involvement}

293 There was no patient and public involvement in this study.

\section{Results}

\section{Effect sizes from primary studies}

\section{Descriptives of primary studies}

297 We analyzed a total of 276,904 treatment effects from 57,162 studies. Studies were published 298 between 1922 to 2019; Psychiatry \& mental health had the largest number of studies $(8,091)$ and 299 Neonatology the smallest $(1,600)$. The median study sample size was $N=91$ (IQR 46-212). The 300 largest median effects (median Pearson's $r$ ) were observed in Anesthesia \& pain $(r=0.23$; IQR $3010.10-0.39)$ and Skin \& wounds ( $r=0.23$; IQR 0.10-0.42), the lowest in public health \& work $(r=$ 302 0.13; IQR 0.05-0.25). For safety outcomes we analyzed 47,466 effects from 13,812 studies. For a 303 detailed description of all the variables from primary studies, see Supplementary Table 3-5. 


\section{Statistically significant effects in primary studies}

305 Generally, we observed more significant effects for efficacy outcomes than for adverse events with 306 the exception of Oncology and in Pregnancy \& childbirth (Figure 2A, Supplementary Table 6). The 307 proportions of significant effects in efficacy and safety outcomes differed among specialties (both $p$ $308<0.0001)$. The largest were $36 \%$ in two specialties, Skin \& wounds (95\%-CI 0.35-0.38) and 309 Anesthesia \& pain (95\%-CI 0.36-0.37); the smallest was $18 \%$ in Neonatology (95\%-CI 0.18-0.19). 310 For safety outcomes, the largest proportion of $29 \%$ was in Oncology (95\%-CI $0.27-0.31)$ and the 311 smallest of 8\% was in Emergency \& trauma (95\%-CI 0.07-0.10). We found no association between 312 the proportion of statistically significant effects versus the median sample size across the medical 313 specialties (Figure 2B). We performed a $p$-curve analysis and found that all the specialties had a 314 right-skewed shape and no inflation of $p$-values close to $p=0.05$ (Supplementary Fig. 1).

\section{Odds for a statistical significant effect in a primary study}

316 We modeled the binary outcome of whether a treatment effect was statistically significant. We 317 found that the odds for a significant result was highest with a 1.9-times increase in Anesthesia \& 318 pain $(\mathrm{OR}=1.93 ; 95 \%$-CI $1.79-2.08)$, and lowest with a $38 \%$ reduction in Pregnancy \& childbirth $319(\mathrm{OR}=0.62 ; 95 \%$-CI $0.58-0.67)$, see Figure 2C and Supplementary Table 7. Primary studies 320 published in the $2 \mathrm{nd}$, 3rd or 4th journal rank quartiles were all associated with higher odds for a 321 statistically significant result (compared to the the 1st quartile); the strongest effect had the top 322 quartile (Q4) that showed a 1.5-fold increase of the odds (OR $=1.52 ; 95 \%$-CI 1.32-1.75). Positive 323 associations with significance were found for $\log _{2}$ study size (OR $=1.15 ; 95 \%$-CI $\left.1.14-1.17\right)$ and $324 \log _{2}$ number of citations per year $(\mathrm{OR}=1.07$; 95\%-CI 1.06-1.08); and negative associations for 325 publication year (per 10 years; OR $=0.94 ; 95 \%$-CI $0.92-0.96$ ) and when a primary study was an $326 \operatorname{RCT}(\mathrm{OR}=0.90 ; 95 \%$-CI $0.86-0.94)$.

\section{Combined effect estimates from meta-analyses}

\section{Descriptives of the meta-analyses}

329 A total of 98,966 random effects meta-analyses were calculated from 4,737 intervention reviews. 330 The largest number of meta-analyses were from Psychiatry \& mental-health $(13,906)$; and the 331 median total sample size in the meta-analyses across studies was $N=523$ (IQR 220-1,395). The 332 combined effects (median Pearson's $r$ ) were largest in Skin \& wounds $(r=0.20$; IQR 0.08-0.37) 
333 and lowest in Pregnancy \& childbirth $(r=0.09$; IQR 0.04-0.20) and neonatology $(r=0.09$; IQR 334 0.04-0.20), see descriptives in Supplementary Table 8.

\section{Statistical significance of the combined effects}

336 The proportion of statistically significant results varied across specialties $(p<0.0001)$, the largest 337 was $49 \%$ in Anesthesia \& pain (95\%-CI 0.47-0.50), and the lowest was $21 \%$ in neonatology 338 (95\%-CI 0.20-0.22), see Supplementary Table 9. We performed a $p$-curve analysis and observed 339 that all the specialties had a right-skewed shape with no inflation of $p$-values close to $p=0.05$ 340 (Supplementary Fig. 2).

\section{Odds for a statistical significant effect in a meta-analysis}

342 We modeled the binary outcome of statistical significance of the combined effects from the 343 meta-analyses (Figure 2D and Supplementary Table 10). We found the highest odds with a 2.5-fold 344 increase in Anesthesia \& pain $(\mathrm{OR}=2.46 ; 95 \%$-CI $1.97-3.06)$ and the lowest with a $43 \%$ reduction 345 in Pregnancy \& childbirth $\left(\mathrm{OR}=0.57\right.$; 95\%-CI 0.47-0.68). The number of studies $\left(\log _{2}\right)$ included 346 in a meta-analyses had 1.6-fold higher odds for a statistically significant result (OR $=1.60 ; 95 \%$-CI 347 1.56-1.65). Positive associations with significance had the $\log _{2}$ sample size (OR $=1.08 ; 95 \%$-CI $3481.07-1.10)$ and the $\log _{2}$ number of citations (OR $=1.04 ; 95 \%$-CI $\left.1.02-1.06\right)$. The journal rank and 349 the review year showed no evidence for an effect.

\section{Small-study effects and publication bias}

\section{Prevalence of small study-effects}

352 A total of 5,534 meta-analyses from 949 Cochrane systematic reviews were assessed that satisfied 353 our inclusion criteria. We found small-study effects in 1,054 (19\%) of the meta-analyses (95\%-CI 354 from $18 \%$ to $20 \%$ ), the distribution of $p$-values is in Figure 3A. Infectious diseases had the highest 355 prevalence which was 35\% (95\%-CI from 0.25 to 0.45$)$ and Genetics \& endocrinology the lowest 356 with $10 \%$ (95\%-CI form 0.06 to 0.17$)$, see Figure 3B.

\section{Modeling small study-effects}

358 We modelled the test statistic ( $t$-score) from the regression-based test for funnel plot asymmetry 359 (Figure 3C and Supplementary Table 11). Infectious diseases (0.34; 95\%-CI 0.02-0.66) and 360 Anesthesia \& pain $(0.30 ; 95 \%$-CI from 0.12 to 0.48$)$ showed larger small-study effects compared to 361 the mean across the specialties; Lungs showed less small-study effects $(-0.19 ; 95 \%$-CI from -0.37 
362 to -0.01$)$. The number of studies $\left(\log _{2}\right)(0.27 ; 95 \%$-CI from 0.22 to 0.33$)$ was associated with 363 stronger small-study effects, the sample size $\left(\log _{2}\right)$ with less $(-0.04 ; 95 \%$-CI from -0.07 to -0.01$)$.

364 Looking at the binary outcome small-study effects (yes/no) we observed similar results. Infectious 365 diseases had a 3-fold increased odds for small-study effects, (OR =3.00; 95\%-CI 1.26-7.16), see 366 Figure 3D and Supplementary Table 12 . The number of studies $\left(\log _{2}\right)$ included in the meta-analyses 367 had 2-fold higher odds for small-study effects (OR = 2.23; 95\%-CI 1.89-2.63).

\section{Small-study effects with probable publication bias}

369 Funnel plots of the meta-analyses with evidence for small-study effects were further assessed. From

370 the 1,054 meta-analyses with small study-effects we identified 214 meta-analyses (20\%) having 371 small study-effects with probable publication bias which was 3.9\% (95\%-CI from 3.4\% to $4.4 \%$ ) of 372 the total of 5,534 meta-analyses included. Examples of evidence for small-study effects versus 373 small-study effects with probable publication bias are shown in Figure 5A and Figure 5B, 374 respectively. The intercepts from the regression-based tests which reflect the magnitude of the bias 375 showed more extreme values for small-study effects with probable publication bias than without 376 (Figure 3E); 99.5\% of these were considered as substantial small-study effects based on the 377 categorization by Lin et al. [25].

378 Overall 378 (40\%) from 949 Cochrane systematic reviews had at least one meta-analysis with 379 small-study effects and $115(12 \%)$ reviews had at least one meta-analysis that showed small-study 380 effects with probable publication bias, see Figure 3F for results per medical specialty.

\section{Adjustment of effect estimates from meta-analyses}

382 Regression-based adjustment of the 1,054 meta-analyses with small-study effects resulted in a 383 reduction of the effects in $99 \%$ of the cases and the median change in the combined effect 384 (Pearsons's $r$ ) was -0.09 (Figure 4A); for Copas selection model the reduction was in $77 \%$ of the 385 cases and the median change was -0.02 (Figure 4B).

386 We applied a mixed-model to all 5,534 included meta-analyses to find associations with effect 387 change after regression-bases adjustment and Copas adjustment (Figure 4C-D). For regression, 388 larger adjustments were required for Infectious diseases (-0.05; 95\%-CI from -0.08 to -0.02) and 389 Anesthesia \& pain $(-0.02 ; 95 \%$-CI from -0.040 to -0.001$)$. Similarly, Copas required larger 
390 adjustments for Infectious diseases (-0.02; 95\%-CI from -0.027 to -0.006) and Anesthesia \& pain 391 (-0.01; 95\%-CI from -0.016 to -0.003). Kidney \& transplant had smaller adjustments; however, this 392 was weak evidence. Furthermore, the sample size $\left(\log _{2}\right)$ was associated with smaller adjustments 393 with both methods, the median SJR with smaller adjustment with Copas, and the number of studies 394 was associated with less adjustment for regression, see Supplementary Table 13-14.

395 Adjustment by regression generally performed a stronger shrinkage of the treatment effects 396 compared the Copas selection model analysis; however, the adjustments were consistent between 397 the two methods across the the medical specialties and across meta-analyses (Figure 5E-F). 398 Compared to the regression-based method Copas applied zero or near-zero adjustment in a number 399 of cases.

\section{Change of evidence for treatment effects}

401 The change of evidence via Bayes factor bounds in 5,534 meta-analyses was assessed by 402 comparing the strength of the evidence after adjustment. The adjustment of the effects changed the 403 overall evidence in many meta-analyses when effects were adjusted with regression but not so with 404 Copas. The proportion of meta-analyses with very strong or decisive evidence for a treatment effect 405 decreased from $32 \%$ down to $7 \%$ (regression) but only from $32 \%$ to $31 \%$ for Copas adjustments, 406 see Figure 5G.

\section{${ }_{407}$ Discussion}

408 Here, we found that 19\% from 5,534 large meta-analyses ( $\geq 10$ studies) have evidence for 409 small-study effects demonstrating that smaller studies reported larger effects which affected $40 \%$ of 410 the Cochrane systematic reviews. Most evidence for small-study effects was found in infectious 411 diseases which also required larger adjustment compared to other specialties. The number of 412 studies in a meta-analysis was strongly associated with small-study effects indicating that more 413 studies improve the statistical power to detect bias. It is striking that our result were highly 414 consistent with the previously reported prevalence of asymmetry in 19\% from a total of 366 415 meta-analyses [15]; we applied exactly the same inclusion criteria for the meta-analyses but the 416 main differences were our 15-times larger dataset and that we also considered continuous outcomes 417 which suggests a broder generalizability of our results. 
418 Systematic assessment of funnel plots suggested that publication bias may not be the main driver 419 behind small-study effects. Only about 4\% of the 5,534 meta-analyses demonstrated small-study 420 effects with probable publication bias. It is worth noting that regression-based methods do not take 421 into account the statistical significance of the study effects. Therefore, other reasons for smaller 422 studies reporting larger effects must be considered: confirmation bias, lower quality studies, 423 selection bias in the treatment groups, outcome switching, post-hoc searches for statistical 424 significance, or incomplete reporting of statistically non-significant results [40]. However, all these 425 biases have the same effect on meta-analyses: they all lead to an exaggeration of the combined 426 treatment effect.

427 Regression-based adjustment of meta-analyses changed the evidence of the treatment effects in 428 many cases from decisive to weak while Copas performed a more consevative adjustment with a 429 majority of meta-analyses that received no or near zero adjustment. The two methods are 430 fundamentally different: while regression is based on funnel-plot asymmetry, Copas analysis 431 directly models publication bias and the selection process. Our further assessments of funnel-plots 432 suggested that small-study effects with probable publication bias is much less common and is more 433 in line with the adjustments from Copas analysis. On the other hand regression-based methods tend 434 to overcorrect in some situations, for example, when the adjusted effect turned smaller than the 435 smallest effect reported in a study. This is not in line with an often cited report [21]; but this report 436 is based on a single meta-analysis which cannot be generalized. Future studies may apply shrinkage 437 methods [41] to regression-based adjustments.

438 We found that some medical specialties were more likely to report a statistically significant effect, 439 for example in Anesthesia \& pain and Skin \& wounds while others were less likely to do so, for 440 example Pregnancy \& childbirth and Neonatology. These findings were even confirmed when 441 looking at combined effects from meta-analyses. Reasons for this could be a higher number of true 442 effects in a field, varying statistical power between fields, or differences in reporting of statistically 443 significant versus non-significant results. We replicated previous findings that studies with a 444 statistically significant effect were more likely to be published in prestigious journals [8] and also 445 received a higher number of citations [9,42] which can indeed contribute to publication bias [43]. 446 We also showed that when studies were of higher quality, such as an RCT, they were less likely to 447 report a statistically significant effect. It is highly problematic when studies that report more 448 extreme results become more visible in prestigious journals instead of studies of the highest quality. 
449 Our study had some limitations. First, regression-based methods have been criticised to have a lack 450 of statistical power [44] and there are also other situations where regression methods (and also 451 selection models) may fail: large heterogeneity, no significant studies, or all studies are of similar 452 sizes. Therefore, we followed available guidelines for inclusion criteria of suitable meta-analyses $453[14,15]$. We only included 5,534 (5.6\%) compared to the 98,966 meta-analyses; most were 454 excluded because the number of studies was smaller than ten. However, this selection of 455 meta-analyses may represent the more relevant medical interventions where many studies have 456 been conducted. In some cases our meta-analyses do not exactly correspond to the ones performed 457 in Cochrane Systematic Reviews as these also include unpublished findings; we excluded these as 458 our aim was to assess publication bias when unpublished data is not considered. Altogether, 459 systematic reviewers considered unpublished studies in 115 (11\%) of the 1,054 meta-analyses with 460 small-study effects, and in $26(12 \%)$ of the 214 meta-analyses that have small-study effects with 461 probable publication bias. It seems that finding unpublished evidence is limited.

462 Around half of systematic reviews, in some specialties even less, do not evaluate publication bias 463 and should do so more often $[45,46]$. However, our findings suggest that large, potentially 464 exaggerated effects due to small studies give rise to greater concern than publication bias. 465 Advanced funnel plots with contours [47] and regression based tests are helpful to identify these 466 problems but are not specific to publication bias alone, and novel and innovative methods may 467 prove to be more suitable [48]. Investigators should also provide individual patient data (IPD) to 468 combat publication bias and other biases in the future [49].

\section{${ }_{469}$ Data Availability}

470 All data used in this study are openly available from the Cochrane Library

471 (https://www.cochranelibrary.com/), Google Scholar (https://scholar.google.com/) and SCImago 472 (https://www.scimagojr.com/). Data are also available from the corresponding authors upon 473 reasonable request. 


\section{${ }_{474}$ Code Availability}

475 Software in $\mathrm{R}$ to fetch the data from the Cochrane Library and Google Scholar, as well as the 476 analysis pipeline to process and analyze the data and produce all the figures, tables and the 477 supplementary information is available at osf.io/uv397.

\section{${ }_{478}$ References}

4791 Rothstein HR, Sutton AJ, Borenstein M. Publication bias in meta-analysis. Wiley, Chichester 2006.

4802 Egger M, Smith GD. Bias in location and selection of studies. BMJ 1998;316:61-6.

4813 Sterling TD. Publication Decisions and Their Possible Effects on Inferences Drawn from Tests of 482 Significance--Or Vice Versa. J Am Stat Assoc 1959;54:30-4.

4834 Dwan K, Altman DG, Arnaiz JA, et al. Systematic review of the empirical evidence of study 484 publication bias and outcome reporting bias. PLoS One 2008;3:e3081.

4855 Decullier E, Lhéritier V, Chapuis F. Fate of biomedical research protocols and publication bias in 486 France: retrospective cohort study. BMJ 2005;331:19.

4876 Song F, Parekh-Bhurke S, Hooper L, et al. Extent of publication bias in different categories of research 488 cohorts: a meta-analysis of empirical studies. BMC Med Res Methodol 2009;9:79.

4897 Dickersin K, Chan S, Chalmers TC, et al. Publication bias and clinical trials. Control Clin Trials $490 \quad 1987 ; 8: 343-53$.

4918 Easterbrook PJ, Berlin JA, Gopalan R, et al. Publication bias in clinical research. Lancet $492 \quad 1991 ; 337: 867-72$.

4939 Nieminen P, Rucker G, Miettunen J, et al. Statistically significant papers in psychiatry were cited more often than others. J Clin Epidemiol 2007;60:939-46.

49510 DeVito NJ, Bacon S, Goldacre B. Compliance with legal requirement to report clinical trial results on ClinicalTrials.gov: a cohort study. Lancet 2020;395:361-9.

49711 Sterne JA, Gavaghan D, Egger M. Publication and related bias in meta-analysis: power of statistical 498 tests and prevalence in the literature. J Clin Epidemiol 2000;53:1119-29.

49912 Egger M, Davey Smith G, Schneider M, et al. Bias in meta-analysis detected by a simple, graphical test. $B M J$ 1997;315:629-34.

50113 Lau J, Ioannidis JPA, Terrin N, et al. The case of the misleading funnel plot. BMJ 2006;333:597-600.

50214 Sterne JAC, Sutton AJ, Ioannidis JPA, et al. Recommendations for examining and interpreting funnel plot asymmetry in meta-analyses of randomised controlled trials. BMJ 2011;343:d4002.

50415 Ioannidis JPA, Trikalinos TA. The appropriateness of asymmetry tests for publication bias in meta-analyses: a large survey. CMAJ 2007;176:1091-6. 
16 Harbord RM, Egger M, Sterne JAC. A modified test for small-study effects in meta-analyses of controlled trials with binary endpoints. Stat Med 2006;25:3443-57.

17 Higgins JPT, Thomas J, Chandler J, et al. Cochrane Handbook for Systematic Reviews of Interventions. John Wiley \& Sons 2019.

18 Mavridis D, Salanti G. How to assess publication bias: funnel plot, trim-and-fill method and selection models. Evid Based Ment Health 2014;17:30.

19 Carpenter JR, Schwarzer G, Rücker G, et al. Empirical evaluation showed that the Copas selection model provided a useful summary in 80\% of meta-analyses. J Clin Epidemiol 2009;62:624-31.e4.

20 Copas JB, Shi JQ. A sensitivity analysis for publication bias in systematic reviews. Stat Methods Med Res 2001;10:251-65.

21 Moreno SG, Sutton AJ, Turner EH, et al. Novel methods to deal with publication biases: secondary analysis of antidepressant trials in the FDA trial registry database and related journal publications. $B M J$ 2009;339:b2981.

22 Sutton AJ, Duval SJ, Tweedie RL, et al. Empirical assessment of effect of publication bias on meta-analyses. BMJ 2000;320:1574-7.

23 van Aert RCM, Wicherts JM, van Assen MALM. Publication bias examined in meta-analyses from psychology and medicine: A meta-meta-analysis. PLoS One 2019;14:e215052.

24 Kicinski M, Springate DA, Kontopantelis E. Publication bias in meta-analyses from the Cochrane Database of Systematic Reviews. Stat Med 2015;34:2781-93.

25 Lin L, Shi L, Chu H, et al. The magnitude of small-study effects in the Cochrane Database of Systematic Reviews: an empirical study of nearly 30000 meta-analyses. BMJ Evid Based Med 2020;25:27-32.

26 Lin L, Chu H, Murad MH, et al. Empirical Comparison of Publication Bias Tests in Meta-Analysis. $J$ Gen Intern Med 2018;33:1260-7.

27 Shi L, Lin L. The trim-and-fill method for publication bias: practical guidelines and recommendations based on a large database of meta-analyses. Medicine 2019;98:e15987.

28 Hayashino Y, Noguchi Y, Fukui T. Systematic evaluation and comparison of statistical tests for publication bias. $J$ Epidemiol 2005;15:235-43.

29 von Elm E, Altman DG, Egger M, et al. Strengthening the Reporting of Observational Studies in Epidemiology (STROBE) statement: guidelines for reporting observational studies. BMJ 2007;335:806-8.

54434 Schwarzer G, Carpenter JR, Rücker G. metasens: Advanced Statistical Methods to Model and Adjust 
54635 Rücker G, Schwarzer G, Carpenter JR, et al. Treatment-effect estimates adjusted for small-study effects via a limit meta-analysis. Biostatistics 2011;12:122-42.

54836 Rücker G, Carpenter JR, Schwarzer G. Detecting and adjusting for small-study effects in meta-analysis. Biom J 2011;53:351-68.

55037 Sellke T, Bayarri MJ, Berger JO. Calibration of $\rho$ Values for Testing Precise Null Hypotheses. Am Stat $551 \quad 2001 ; 55: 62-71$.

55238 Held L, Ott M. On p-Values and Bayes Factors. Annu Rev Stat Appl 2018;5:393-419.

55339 Bates D, Mächler M, Bolker B, et al. Fitting Linear Mixed-Effects Models Using lme4. Journal of $554 \quad$ Statistical Software 2015;67:1-48.

55540 Chan A-W, Altman DG. Identifying outcome reporting bias in randomised trials on PubMed: review of publications and survey of authors. BMJ 2005;330:753.

55741 Copas JB. Using regression models for prediction: shrinkage and regression to the mean. Stat Methods Med Res 1997;6:167-83.

55942 Unger JM, Barlow WE, Ramsey SD, et al. The Scientific Impact of Positive and Negative Phase 3 $560 \quad$ Cancer Clinical Trials. JAMA Oncol 2016;2:875-81.

56143 De Oliveira GS Jr, Chang R, Kendall MC, et al. Publication bias in the anesthesiology literature. Anesth Analg 2012;114:1042-8.

56344 Furuya-Kanamori L, Xu C, Lin L, et al. P value-driven methods were underpowered to detect publication bias: analysis of Cochrane review meta-analyses. J Clin Epidemiol 2020;118:86-92.

56545 Hedin RJ, Umberham BA, Detweiler BN, et al. Publication Bias and Nonreporting Found in Majority of Systematic Reviews and Meta-analyses in Anesthesiology Journals. Anesth Analg

567 2016;123:1018-25.

56846 Herrmann D, Sinnett P, Holmes J, et al. Statistical controversies in clinical research: publication bias evaluations are not routinely conducted in clinical oncology systematic reviews. Ann Oncol

57147 Peters JL, Sutton AJ, Jones DR, et al. Contour-enhanced meta-analysis funnel plots help distinguish publication bias from other causes of asymmetry. J Clin Epidemiol 2008;61:991-6.

48 Mathur MB, VanderWeele TJ. Sensitivity analysis for publication bias in meta-analyses. J R Stat Soc C 2020;13:775.

57549 Ahmed I, Sutton AJ, Riley RD. Assessment of publication bias, selection bias, and unavailable data in 


\section{${ }_{577}$ Author contributions}

578 S.S. and L.H. designed the study. S.S. developed software and collected the data. S.S. and G.K. 579 analysed the data. S.S. wrote the first draft of the manuscript and all authors commented on 580 subsequent versions.

\section{${ }_{581}$ Funding}

582 S.S. received funding from $\mathrm{SfwF}$ (Stiftung für wissenschaftliche Forschung an der Universität 583 Zürich; grant no. STWF-19-007).

\section{${ }_{584}$ Competing interests}

585 The authors declare no competing interests.

\section{${ }_{586}$ Patient consent for publication}

587 Not required.

\section{Acknowledgements}

589 We thank Maya B. Mathur for commenting on the protocol and Philip Heesen for commenting on 590 an earlier draft of the manuscript. We thank Cochrane for providing the data and their tremendous 591 efforts to synthesize evidence in health care.

\section{${ }_{592}$ Additional information}

593 Supplementary Information accompanies this paper at osf.io/dtqs5. The study protocol is available 594 at osf.io/3a28k. 


\section{${ }_{595}$ Figure Captions}

\section{a Selection of systematic reviews} Records identified through the Cochrane Library
- intervention reviews $(n=7,931)$

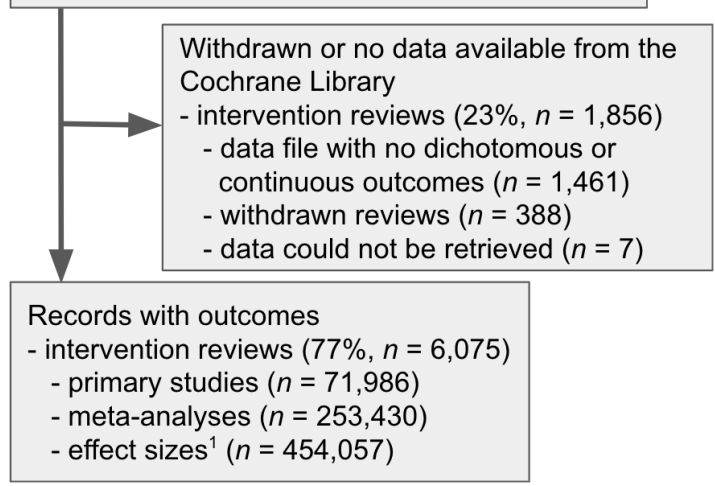

C Selection of combined treatment effect estimates from meta-analyses

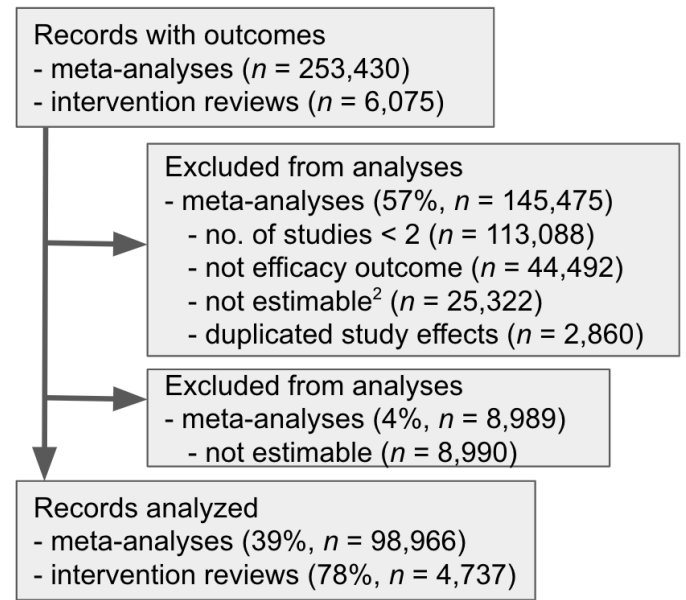

\section{b Selection of treatment effect from primary studies}

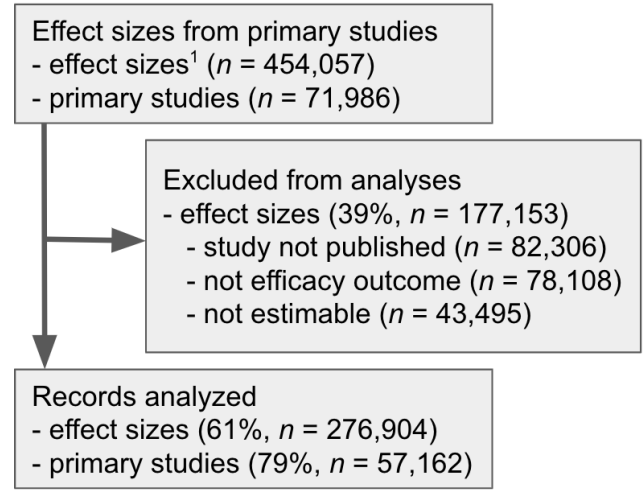

d Inclusion of meta-analyses for asymmetry and publication bias tests

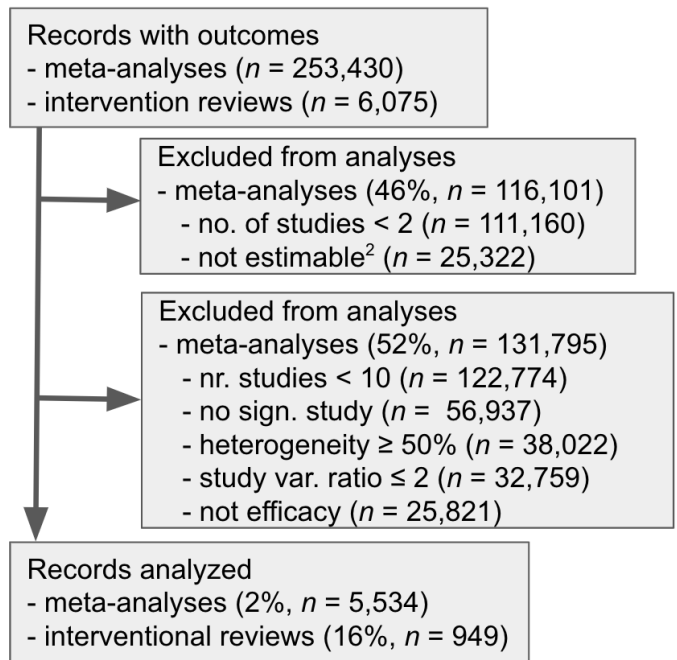

${ }^{1}$ Effect sizes unique in a medical specialty; the effect sizes unique across specialties were $n=453,397$ ${ }^{2}$ Not estimable as specified in the CDSR

596 Figure 1 Selection process from the Cochrane Database of Systematic Reviews (CDSR). (A) Data from $59777 \%$ of the systematic reviews were retrieved. Selection of treatment effects from primary studies (B) and 598 selection of meta-analyses (C-D). 
a

Prevalence of stat. significance $\mathbf{b}$ among 276,904 effects

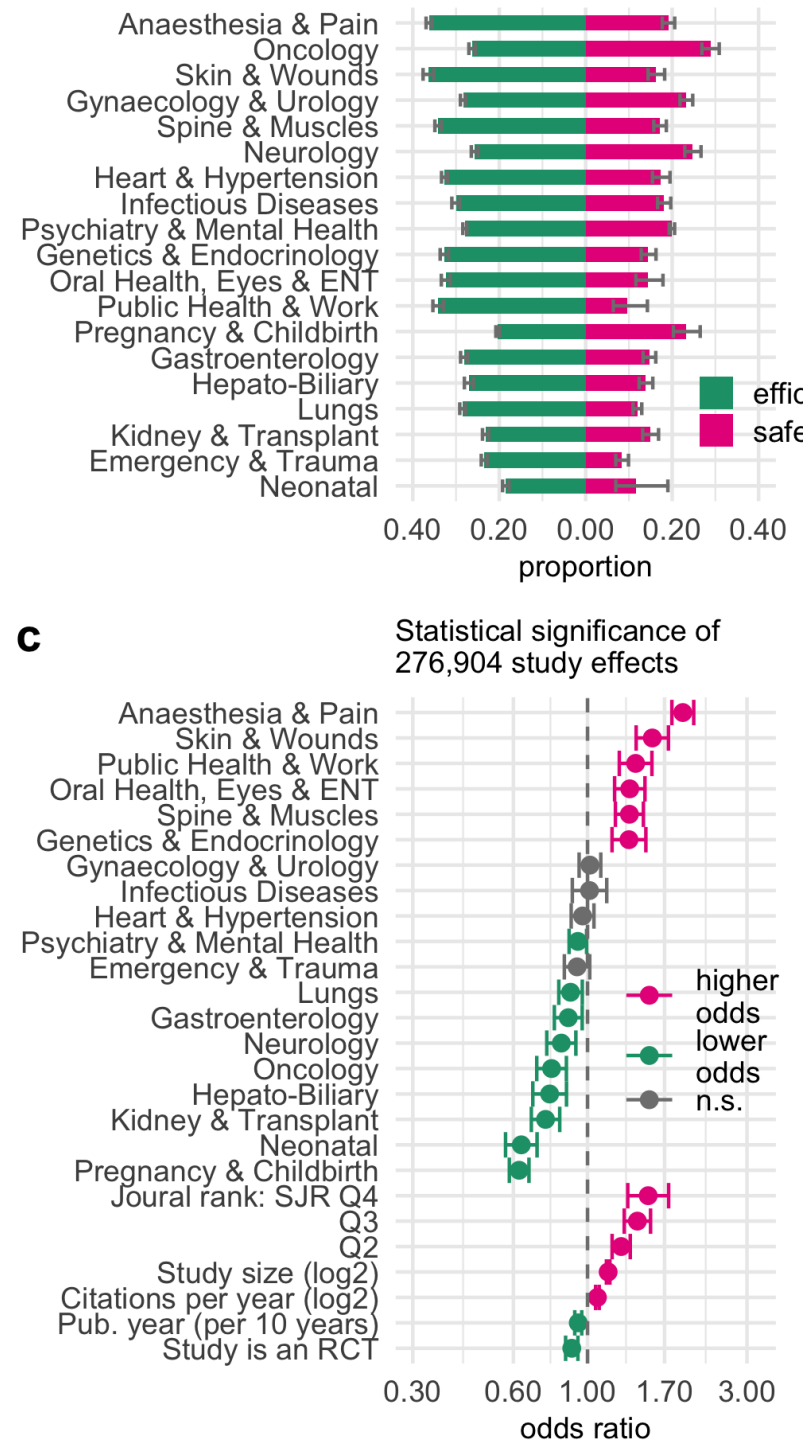

Study sample size versus reported stat significant effects per specialty

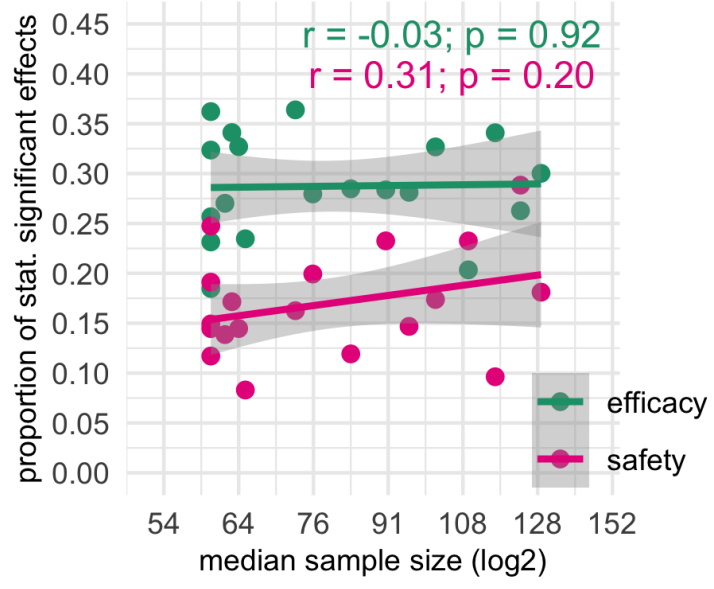

Statistical significance of 98,966 meta-analyses

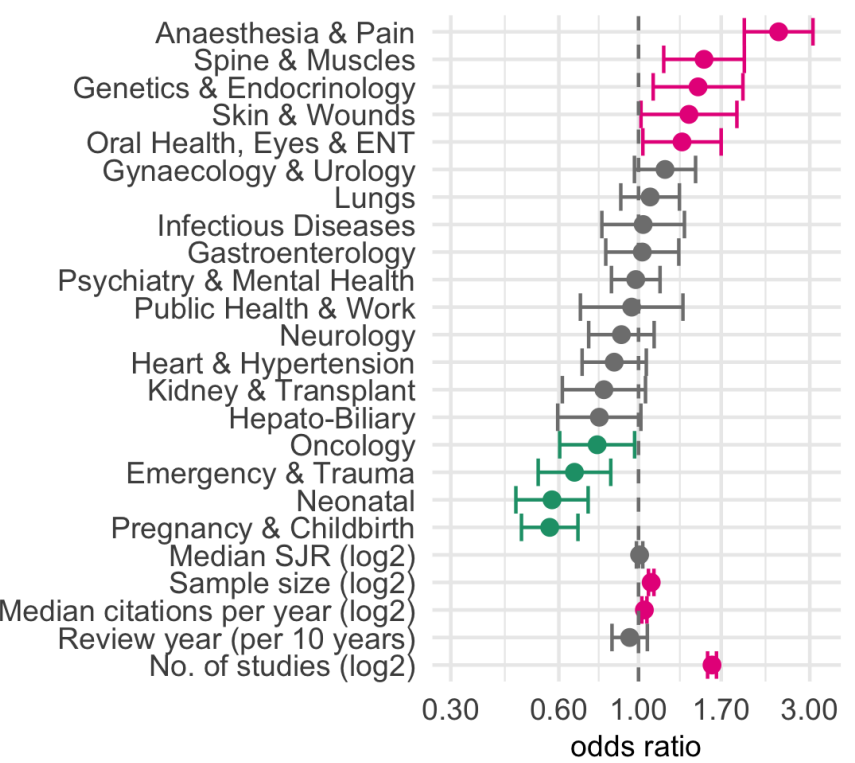

599 Figure 2 Statistically significant results across medical specialties. (A) Proportion (with 95\%-Cl) of reported

600 statistically significant effects in published studies for efficacy and safety outcomes. (B Relationship between

601 the proportion of statistically significant effects and the median sample size. Odds ratios (with 95\%-Cl) for a

602 statistically significant effect in a primary study (C) and in a combined effect from a meta-analysis (D). 
a p-values from regression-based tests of 5,534 meta-analyses

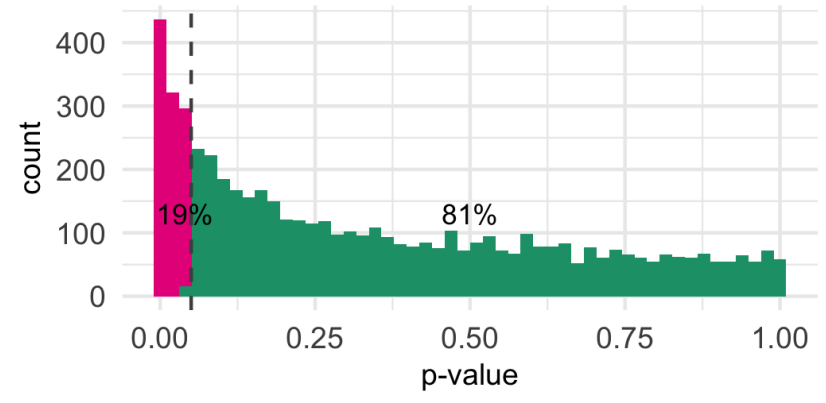

C

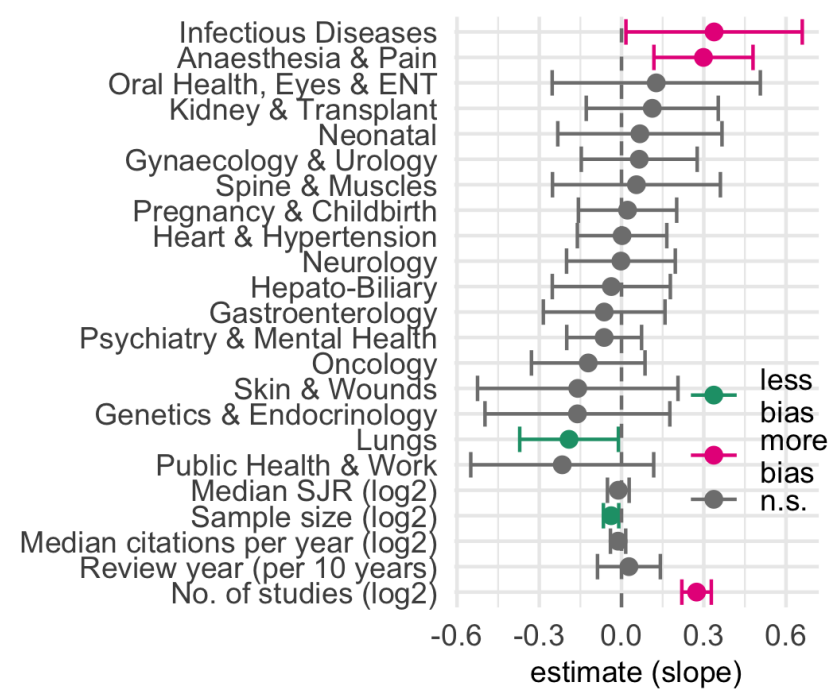

b Prevalence of small-study effects in 5,534 meta-analyses

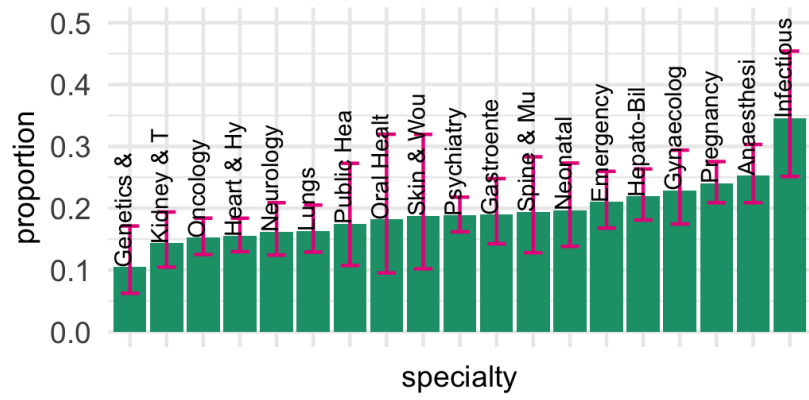

Small-study effects (yes/no) in 5,534 meta-analyses

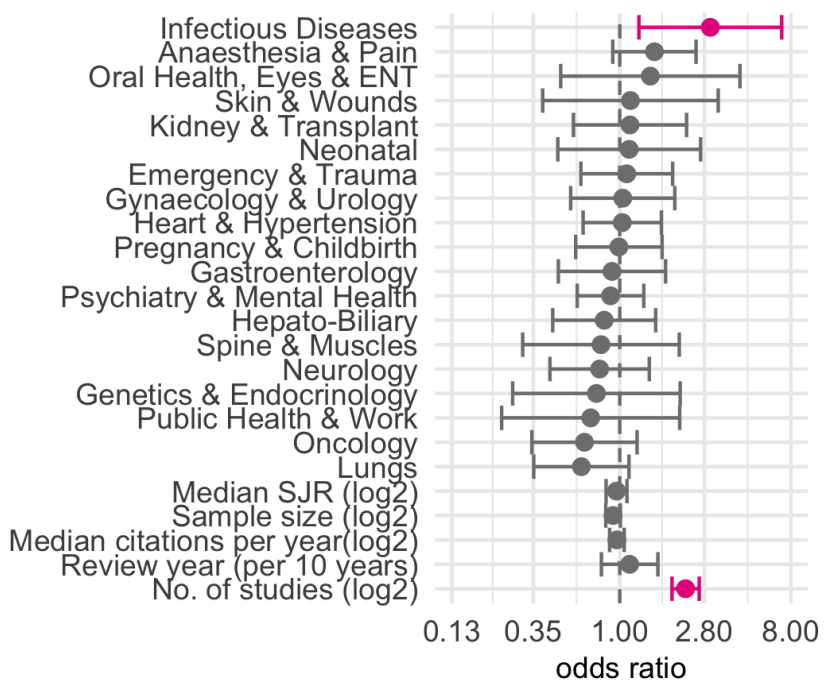

e Intercepts from 5,534 regression-based tests versus bias categories

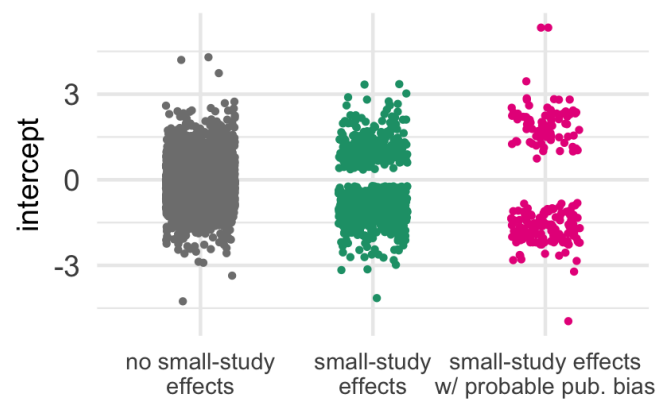

f

Absolute number of Cochrane systematic reviews with at least one meta-analysis affected by bias

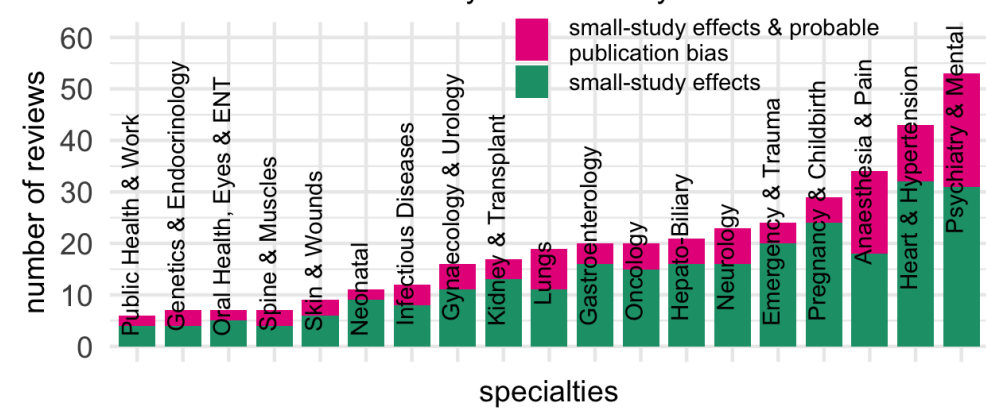

603 Figure 3 Small-study effects in meta-analyses across medical specialties. (A) Distribution of $p$-values from

604 regression-based tests, $p$-values $<0.05$ (one-sided) were considered as evidence for small-study effects in

605 meta-analyses (red). (B) Prevalence (with 95\%-Cl) of small-study effects across the medical specialties. (C)

606 Regression estimates (with $95 \%$-Cl) for small-study effects with the outcome $t$-scores from regression-based

607 tests. (D) Same but for a binary outcome (small-study effects yes/no). (E) Distribution of intercepts

608 (magnitude of small-study effects) from regression-based test. (F) Number of systematic reviews affected by

609 bias. 
a Distribution of effect changes from 5,534

a meta-analyses after adjustment (regression)

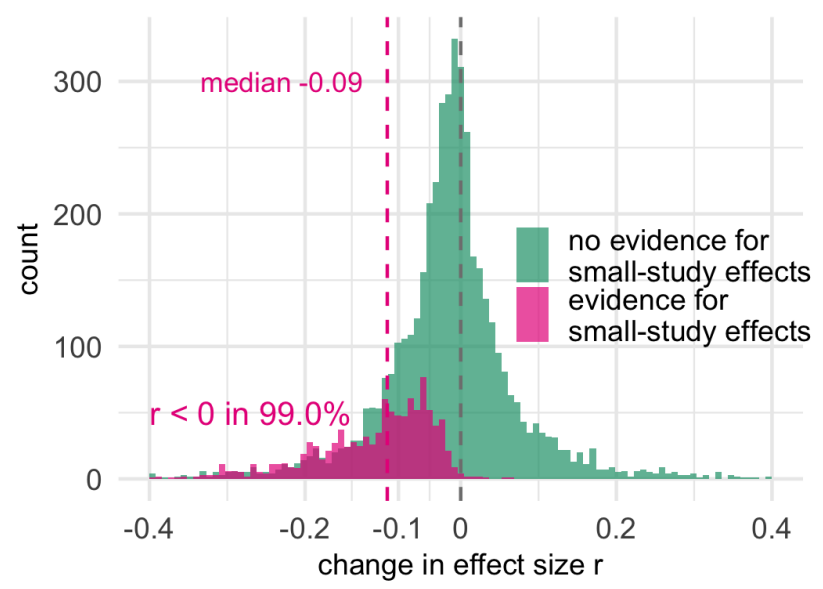

Change in effect (regression)

C Genetics \& Endocrinology Kidney \& Transplant

Public Health \& Work

Pregnancy \& Childbirth Neonatal Neurology

Oral Health, Eyes \& ENT Gynaecology \& Urology Psychiatry \& Mental Health Emergency \& Trauma Spine \& Muscles

Heart \& Hypertension Gastroenterology Gastroenterology
Anaesthesia \& Pain Skin \& Wounds Infectious Diseases Median SJR ( $\log 2)$ Sample size $(\log 2$ Med. citations per year $(\log 2$ Review year (per 10 years) No. of studies $(\log 2)$

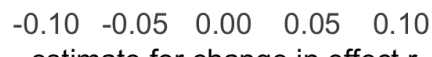
estimate for change in effect $r$

e

\section{f}

Adjustments by regression vs. Copas selection model for 19 medical specialties
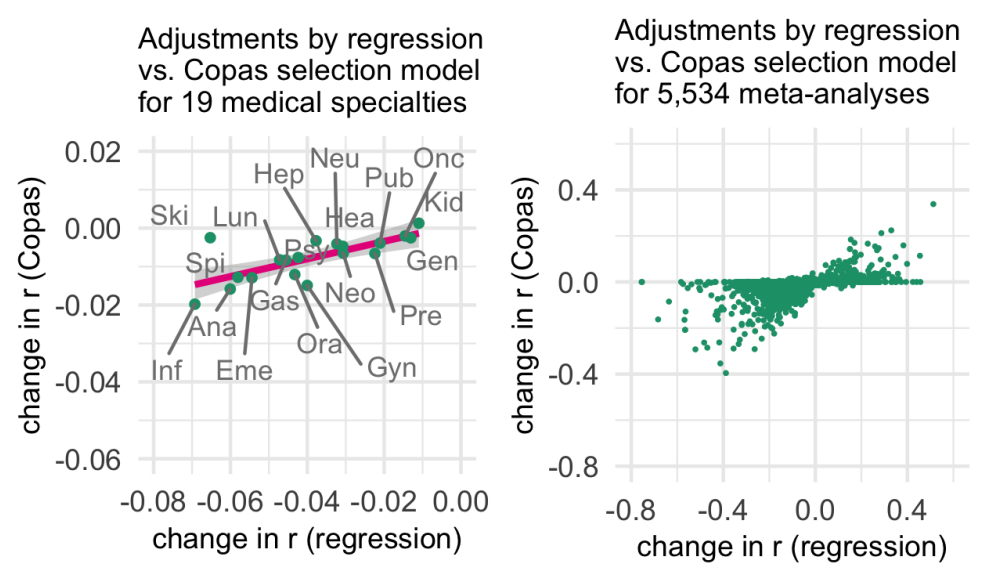

b Distribution of effect changes from 5,534

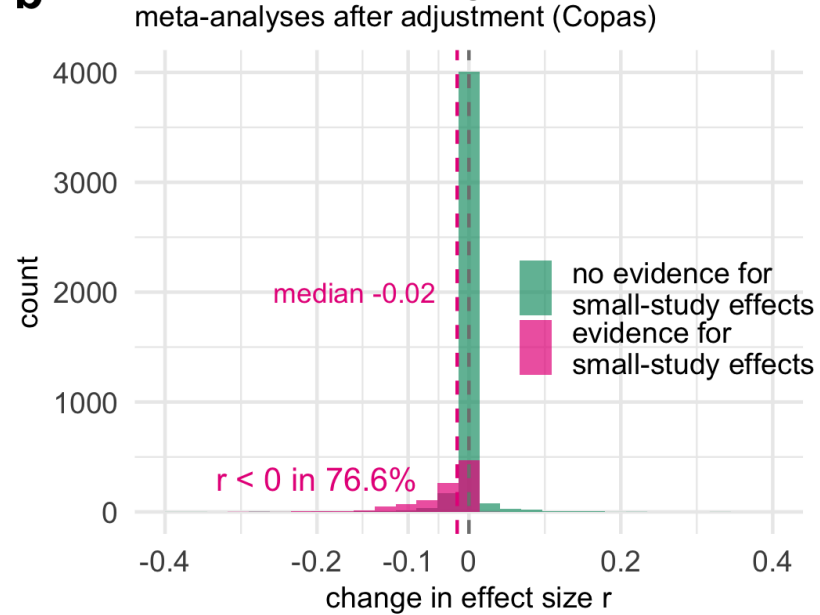

Change in effect (Copas)

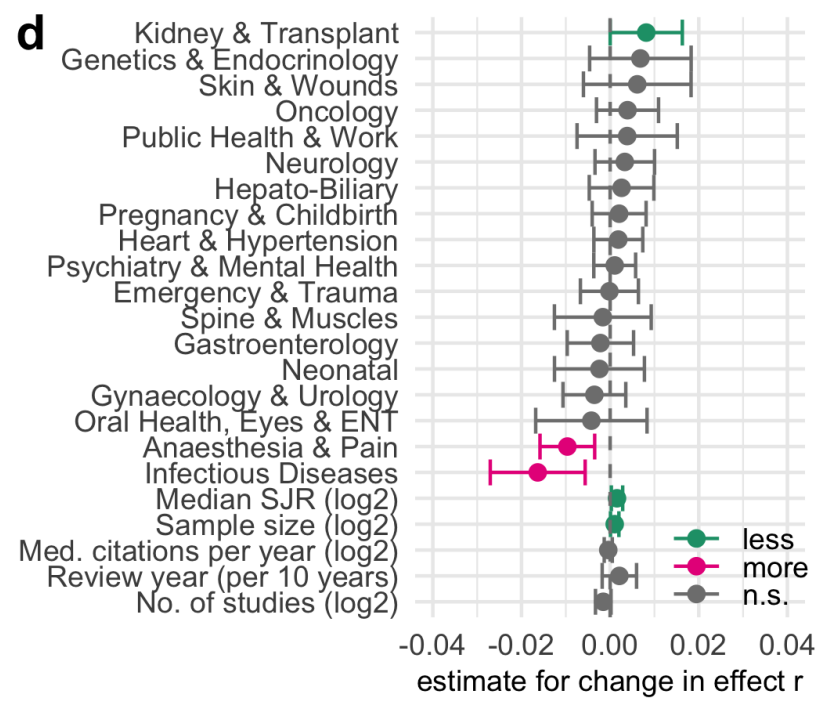

g

Strengh of evidence favoring the treatment effect in 5,534 meta-analyses
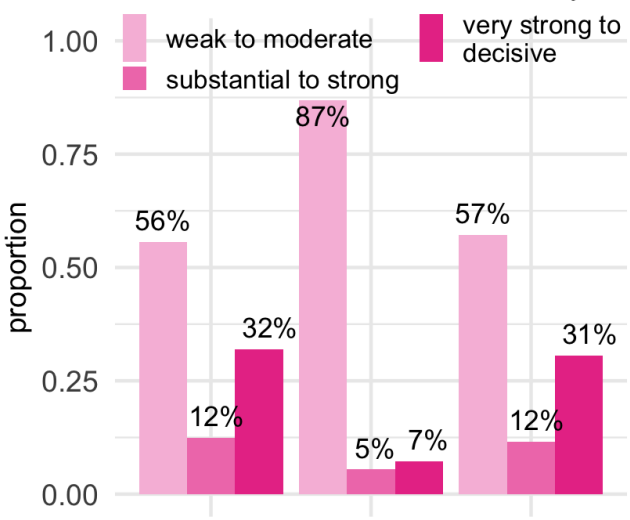

random-effects $M A$
adjusted by regression
adjusted by Copas

610 Figure 4 Adjustment of treatment effects. (A) Distribution of effect changes (Pearson's $r$ ) from

611 meta-analyses after adjustment by regression; (B) same for Copas adjustment. (C) Variables associated

612 with effect changes, negative estimates reflect shrinkage of effects; (D) same for Copas adjustment. (E)

613 Adjustment by regression versus Copas across specialties; (F) same across all meta-analysis. (G) Strengths

614 of evidence against the null of no treatment effect before (random effects meta-analysis) and after

615 adjustment (regression and Copas). 
a
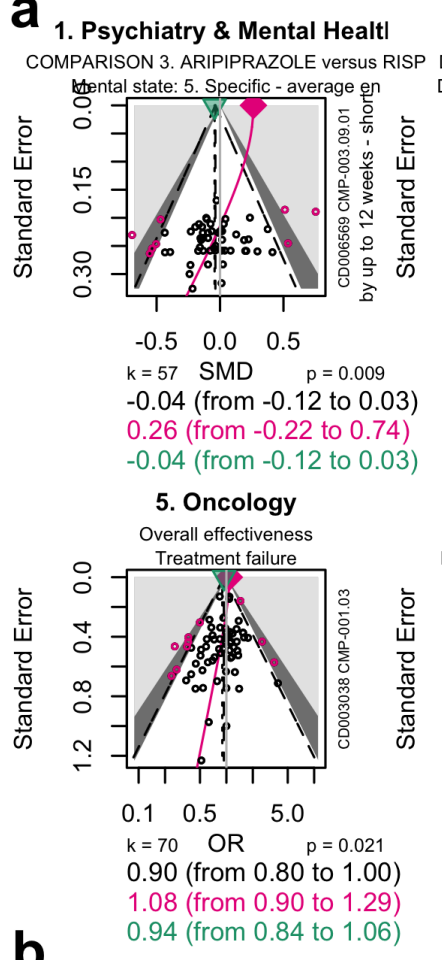

b

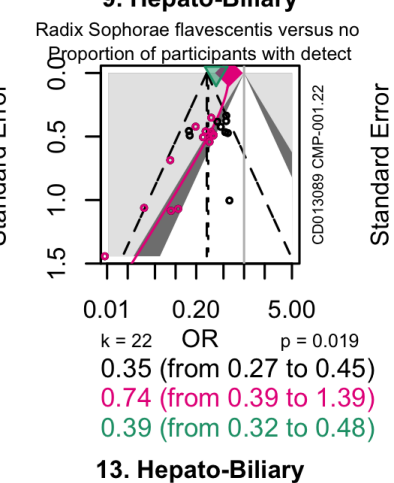

Radix Sophorae flavescentis versus no

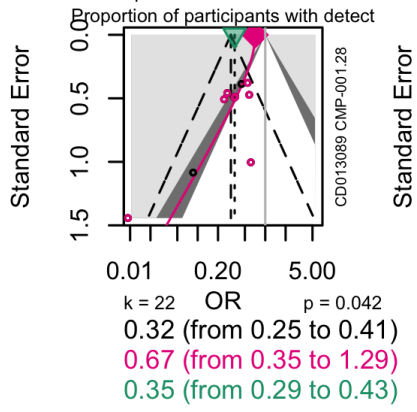

2. Neonatal

Death or bronchopulmonary dysplasia (B Death or BPD at 36 weeks' postmenstrua

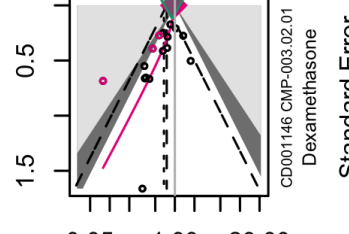

$\begin{array}{lll}0.05 & 1.00 \quad 20.00\end{array}$

$k=16 \quad$ OR $\quad p=0.011$

0.68 (from 0.53 to 0.88 )

0.97 (from 0.67 to 1.38 )

0.91 (from 0.75 to 1.10 )

6. Oral Health, Eyes \& ENT

$50 \%$ pain relief using pain intensity $\mathrm{m}$

a aracetamol versus placebo: number of

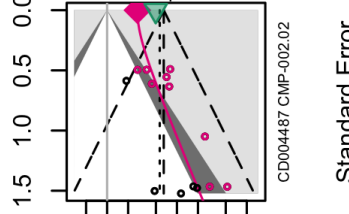

$\begin{array}{lll}0.5 & 5.0 & 50.0\end{array}$

$\mathrm{k}=14$ OR $\mathrm{p}=0.0003$

5.67 (from 3.70 to 8.70 )

2.71 (from 1.09 to 6.73 )

4.96 (from 3.41 to 7.22 )

10. Anaesthesia \& Pain

Rofecoxib $50 \mathrm{mg}$ versus placebo

Barticipants with at least $50 \%$ pain re

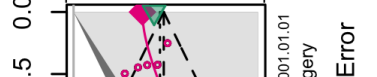

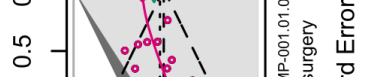
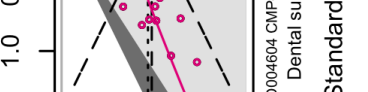

우

$\begin{array}{llll}1 & 5 & 20 & 200\end{array}$

$\mathrm{k}=17$ OR $\quad \mathrm{p}=0.012$

16.28 (from 11.56 to 22.94 )

9.24 (from 5.12 to 16.68 )

13.47 (from 9.39 to 19.32 )

14. Psychiatry \& Mental Healt

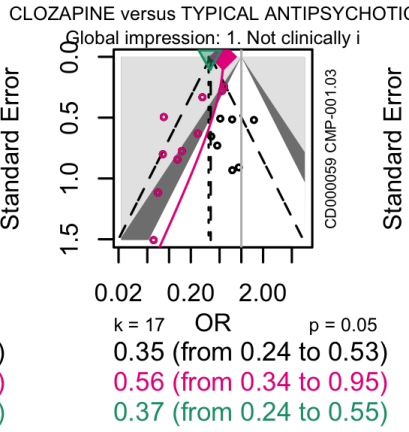

3. Oncology

Participants receiving red blood cell

Participants receiving red blood cell

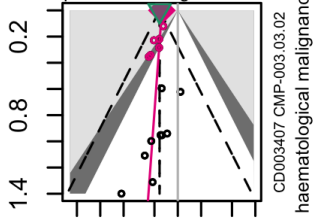

$\begin{array}{lll}0.05 & 0.50 \quad 5.00\end{array}$

$k=15$ OR $p=0.009$

0.58 (from 0.50 to 0.68 )

0.62 (from 0.49 to 0.80 )

0.58 (from 0.50 to 0.68 )

7. Neonatal

Phenobarbital versus control

Severe intraventricular haemorrhage

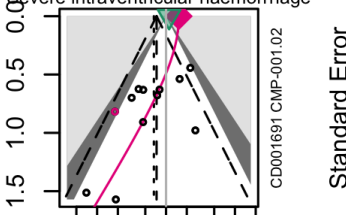

$\begin{array}{lll}0.05 & 0.50 \quad 5.00\end{array}$

$k=12$ OR $\quad p=0.005$

0.67 (from 0.38 to 1.16 )

1.54 (from 0.44 to 5.38 )

1.11 (from 0.60 to 2.04 )

\section{Pregnancy \& Childbirth}

Immediate or Early skin-to-skin versus

Bgastfeeding 1 month to 4 months post

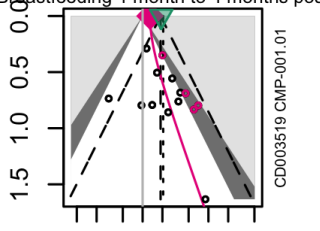

$\begin{array}{lll}0.1 & 1.0 & 10.0\end{array}$

$\mathrm{k}=14 \quad$ OR $\quad \mathrm{p}=0.034$

2.06 (from 1.38 to 3.07 )

1.30 (from 0.62 to 2.71 )

1.92 (from 1.27 to 2.88 )

15. Lungs

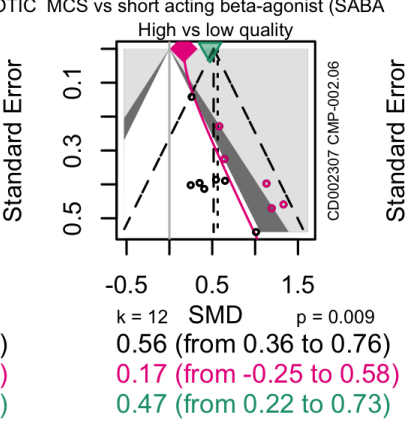

4. Psychiatry \& Mental Healt|

Mirtazapine versus SSRIs

Primary outcome (response) at end of $t$
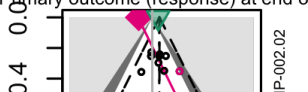

$\infty$

@

ヘ

$\begin{array}{llll}1 & 1 & 1\end{array}$

$k=11 \quad$ OR $\quad p=0.015$

1.20 (from 1.00 to 1.43 )

0.71 (from 0.41 to 1.22 )

1.16 (from 0.97 to 1.39 )

8. Hepato-Biliary

Intervention versus control

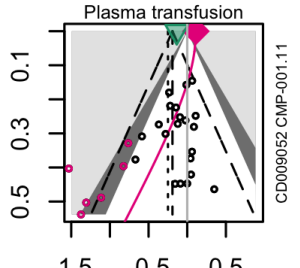

$\begin{array}{lll}-1.5 & -0.5 & 0.5\end{array}$

$k=27$ SMD $\quad p=0.003$

-0.25 (from -0.40 to -0.10 )

0.11 (from -0.18 to 0.40 )

-0.14 (from -0.27 to 0.00 )

12. Hepato-Biliary

Antibiotics versus no intervention/pla

Bacterial infections according to the

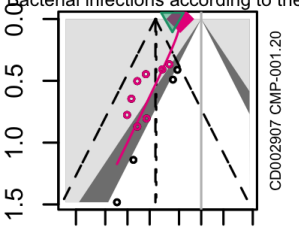

$\begin{array}{lll}0.02 & 0.20 & 2.00\end{array}$

$k=12$ OR $\quad p=0.0007$

0.25 (from 0.18 to 0.34 )

0.52 (from 0.22 to 1.24 )

0.41 (from 0.28 to 0.59 )

16. Anaesthesia \& Pain

Subgroup by year of study

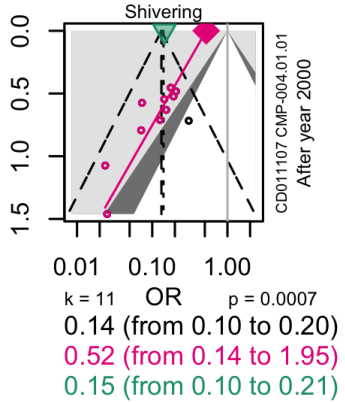

616 Figure 5 Enhanced funnel plots. Randomly picked examples of funnel plots with small-study effects (A); and

617 small-study effects with probable publication bias (B). Small black circles are studies; statistically significant

618 ones in red. The shades of gray are different levels of statistical significance (gray is $p<0.05$, light gray is $p$

619 < 0.01). The gray vertical line is the null, the dashed line the fixed effects, the dotted line the random effects

620 estimate. The red diamont (regression) and green rectangle (Copas) are the adjusted effects, also as

621 numbers (with 95\%-Cl) at the bottom (unadjusted random effects estimate in black). The $p$-value is from the

622 asymmetry test by regression, $\mathrm{k}$ the number of studies. Above the funnel plots are the medical specialty, the

623 outcome and comparison name; on the right the review id, meta-analysis id and the subgroup name. 